\title{
USO DE PRÁCTICAS CLÍNICAS DURANTE EL EMBARAZO, PARTO, PUERPERIO Y RECIEN NACIDO, EN HOSPITALES PÚBLICOS DE MANIZALES - COLOMBIA, 2005
}

\section{Using guides for clinical practice during pregnancy, delivery and postpartum and for the newborn in public hospitals in Manizales, Colombia, 2005}

Fernando Arango, M.D. *, Joaquín Guillermo Gómez, M.D., M.Sc**, John Jairo Zuleta, M.Sc***

Recibido: septiembre 9/2005 - Revisado: octubre 10/2005 - Aceptado: noviembre 17/2005

\section{RESUMEN}

Se evaluó la frecuencia de uso de prácticas comprobadas científicamente como beneficiosas, inefectivas o perjudiciales, en el cuidado del embarazo, trabajo de parto, parto, puerperio y periodo neonatal, en tres centros públicos de atención en salud de la ciudad de Manizales. Algunas prácticas clínicas que se han demostrado benéficas, aún no se han implementado o no se utilizan en forma rutinaria; tal es el caso del uso del carné materno perinatal, el tamizaje para sífilis durante la gestación, el diligenciamiento del partograma, la terminación de la gestación luego de las 41 semanas para reducir la mortalidad perinatal, el apoyo continuo a la mujer durante el trabajo de parto y parto, el manejo activo del alumbramiento para prevenir la hemorragia postparto, la lactancia materna exclusiva y el pinzamiento del cordón umbilical después del primer minuto. De igual forma, otras prácticas que se han demostrado inefectivas e incluso perjudiciales, todavía se siguen

* Pediatra Neonatólogo, Profesor Asistente Universidad de Caldas Correo electrónico: farango@telesat.com.co

** Gineco-Obstetra, Magister en Epidemiología Clínica, Profesor Asistente Universidad de Antioquia.

*** Gineco-Obstetra, Magister en Epidemiología Clínica, Profesor Asociado Universidad de Antioquia. utilizando, como es el caso del enema evacuante durante el trabajo de parto, la posición en decúbito dorsal (supino) en el periodo expulsivo del parto y la restricción del contacto madre-hijo en los recién nacidos sanos. Debe implementarse una política restrictiva de episiotomía, evitando realizarla de manera rutinaria en las primíparas.

Palabras clave: prácticas clínicas, embarazo, parto, recién nacido.

\section{SUMMARY}

The frequency of use of some forms of care which have been scientifically tested as being beneficial, likely to be harmful or ineffective during pregnancy, labor, puerperium and neonatal periods were evaluated in three of Manizales' public health centres. Some beneficial practices have not yet been implemented or are not being used systematically, such as giving women their own case notes to carry during pregnancy, congenital syphilis screening during pregnancy, using a partograph, inducing labor for improving the outcome of delivery beyond term, continuous support for women during childbirth, active management during the third stage of labor, exclusive breastfeeding and delayed cord 
clamping. Other ineffective or harmful practices are still being used, such as giving enemas during labor, using a supine maternal position during labor and restricting early contact with mothers having healthy newborn infants. A restrictive episiotomy policy should be implemented in nulliparous and primiparous births.

Key words: clinical practices, pregnancy, labor, infant, newborn.

\section{INTRODUCCIÓN}

En América Latina y el Caribe, una proporción significativa de los cuidados que se administran a las mujeres y a los recién nacidos durante el período prenatal, el parto y el puerperio están científicamente comprobados como inefectivos o perjudiciales, y buena parte de los comprobados como beneficiosos no son utilizados rutinariamente. ${ }^{1}$ También son de uso rutinario una serie de prácticas sobre las cuales no existe evidencia científica acerca de sus efectos, por lo tanto, no se conoce si son benéficas o perjudiciales para la salud o son simplemente inefectivas y costosas. En la Clínica Villapilar de la Empresa Social del Estado Rita Arango Alvarez del Pino, y las Clínicas de la Enea y la Asunción de la Asociación de Servicios Básicos de Salud, Assbasalud, de la ciudad de Manizales no se conocía la frecuencia real de uso de dichas prácticas, requisito importante para el desarrollo de guías de práctica clínica basadas en la evidencia, con el fin de estimular el uso de las prácticas benéficas y desestimular el uso de las prácticas inefectivas o perjudiciales. El objetivo de este estudio es describir la utilización de tales prácticas en tres instituciones de diferente grado de complejidad en Colombia.

\section{MATERIALES Y MÉTODOS}

Este es un estudio descriptivo, prospectivo, de corte transversal, que evaluó la frecuencia de uso de prácticas comprobadas científicamente como beneficiosas, inefectivas o perjudiciales, en el cuidado del embarazo, trabajo de parto, parto, puerperio y periodo neonatal, en la E.S.E. Rita Arango Alvarez del Pino - Clínica Villapilar, institución de tercer nivel de complejidad, y en las Clínicas La Asunción y La Enea de Assbasalud, de la Secretaría de Salud y Seguridad Social de la ciudad de Manizales, instituciones del primer nivel de complejidad, y hace parte de una investigación multicéntrica de la Red Colombiana de Centros Asociados al Centro LatinoAmericano de Perinatología y Desarrollo Humano (CLAP/OPS), con el apoyo del Instituto Colombiano para el Desarrollo de la Ciencia y Tecnología "Francisco José de Caldas" (Colciencias).

El trabajo fue aprobado por el comité de ética de las instituciones participantes, por ser una investigación con riesgo menor al mínimo, no se solicitó consentimiento informado. Se garantizó la confidencialidad de las participantes.

Se incluyeron todas las mujeres que tuvieron su parto en las Clínicas Villapilar, La Enea y La Asunción. Se incluyó también a todos los recién nacidos vivos en dichos centros hospitalarios, entre el 1 de julio de 2004 y el 31 de marzo de 2005. La fuente primaria para obtener la información fue la historia clínica materno-perinatal, del Sistema Informático Perinatal (SIP), desarrollado por el CLAP-OPS. No se consideraron criterios de exclusión.

Se definió como parto la culminación del embarazo a una edad gestacional igual o mayor a 24 semanas. Para la edad materna se consideró los años cumplidos al momento de la primera consulta. El nivel de estudios correspondió al número de años completados en el mayor nivel alcanzado (primario, secundario, universitario). El número de controles prenatales considerado fue el total de consultas realizadas por médico o enfermero a las que asistió la madre durante la gestación. El régimen contributivo es el conjunto de normas que rigen la vinculación de los individuos y las familias al Sistema General de Seguridad Social en Salud (SGSSS) de Colombia, cuando se hace a través del pago de una cotización, individual y familiar, o un aporte económico previo financiado directamente por el afiliado o en concurrencia entre éste y su empleador. El régimen 
subsidiado son las normas que rigen el ingreso de las personas (y su núcleo familiar) sin capacidad de pago al SGSSS, cuando esta afiliación se hace a través del pago total o parcial de la unidad de pago por capitación subsidiada con recursos fiscales o de solidaridad. La problación vinculada (no asegurada) son las personas que no tienen capacidad de pago, que se encuentran en proceso de afiliación al régimen subsidiado.

Los criterios para seleccionar las prácticas clínicas fueron los siguientes: a) prácticas demostradas como efectivas por investigaciones clínicas aleatorizadas, para reducir causas directas prevalentes de morbilidad o mortalidad materna y perinatal, o reducir factores de riesgo asociados con ellas; b) prácticas demostradas como efectivas por investigaciones clínicas aleatorizadas, para reducir problemas clínicos o mejorar la satisfacción de las mujeres, y que puedan ser aplicadas a la mayor parte de la población (más del 50\%) de mujeres o neonatos; c) prácticas demostradas como inefectivas por investigaciones clínicas aleatorizadas, para reducir causas de morbilidad o mortalidad materna y perinatal, o factores de riesgo asociados con ellas, y que puedan causar daño o insatisfacción en las mujeres o neonatos.

Se seleccionaron prácticas que cumplieron con al menos uno de los tres criterios. La fuente de selección fueron las tablas de prácticas efectivas, inefectivas o perjudiciales publicadas en el libro A Guide to Effective Care in Pregnancy and Childbirth ${ }^{2} \mathrm{y}$ en la Biblioteca de Salud Reproductiva de la OMS, versión 5. ${ }^{3}$ El proceso de selección contó con la colaboración de los coordinadores de investigación de la Red de Centros Asociados al Centro LatinoAmericano de Perinatología y Desarrollo Humano (CLAP/OPS).

Las prácticas fueron seleccionadas por consenso y clasificadas en cinco grupos: prácticas durante el cuidado de la gestación, prácticas de detección sistemática y diagnóstico, prácticas para el manejo de los problemas del embarazo, prácticas correspondientes a la atención del parto y prácticas correspondientes al cuidado del recién nacido. Las prácticas durante el cuidado de la gestación seleccionadas fueron: que las mujeres lleven un registro de su embarazo para fomentar el sentimiento de que están siendo supervisadas (se tuvo en cuenta el número de mujeres que presentaron el carné materno-perinatal al momento del parto dividido sobre el número de partos de mujeres con al menos un control prenatal), la suplementación con hierro y ácido fólico durante la gestación (número de mujeres con al menos un control prenatal a quienes se les formuló hierro $\mathrm{y} / \mathrm{o}$ ácido fólico dividido sobre el número de partos de mujeres con al menos un control prenatal). Las prácticas de detección sistemática y diagnóstico seleccionadas fueron la detección de sífilis gestacional (número de mujeres con al menos un control prenatal y al menos una determinación de VDRL dividido sobre el número de partos de mujeres con al menos un control prenatal) y la detección de bacteriuria asintomática (número de mujeres con bacteriuria en el parcial de orina dividido sobre el número de partos de mujeres con al menos un control prenatal). Las prácticas para el manejo de los problemas del embarazo seleccionadas fueron: uso de sulfato de magnesio para el tratamiento de la eclampsia (número de partos en mujeres con eclampsia que recibieron sulfato de magnesio dividido sobre el número de partos de mujeres con eclampsia), uso de sulfato de magnesio para el tratamiento de la pre-eclampsia severa (número de partos en mujeres con pre-eclampsia severa que recibieron sulfato de magnesio dividido sobre el número de partos de mujeres con pre-eclampsia severa) y uso de corticoides antenatales para promover la maduración fetal en embarazadas con alto riesgo de parto pretérmino (recién nacidos vivos menores de 37 semanas de gestación cuyas madres hubieran recibido al menos una dosis de corticoides para maduración fetal dividido sobre el número de recién nacidos vivos de menos de 37 semanas de gestación). Las prácticas correspondientes a la atención del parto seleccionadas fueron: uso de enema evacuante durante el trabajo de parto (número de mujeres con parto vaginal que recibieron enema evacuante divi- 
dido sobre el número de mujeres con parto vaginal), rasurado perineal de rutina para la preparación del parto (número de mujeres con parto vaginal que recibieron rasurado perineal dividido sobre el número de mujeres con parto vaginal), diligenciamento del partograma durante el trabajo de parto y parto (número de mujeres con parto vaginal a quienes se les diligenció el partograma dividido sobre el número de mujeres con parto vaginal), posición de decúbito dorsal (supino) en el periodo expulsivo del parto (número de mujeres con parto vaginal en posición supina dividido sobre el número de mujeres con parto vaginal), episiotomía rutinaria en primíparas (número de partos en primíparas con episiotomía dividido sobre el número de partos vaginales en primíparas), terminación de la gestación luego de 41 semanas para reducir la mortalidad perinatal (número de partos con edad gestacional $\geq 41$ semanas con inicio inducido o cesárea electiva dividido sobre el número de partos con edad gestacional $\geq$ 41 semanas), apoyo continuo para la mujer durante el trabajo de parto (número de mujeres con acompañante durante todo el trabajo de parto dividido sobre el número de mujeres con parto vaginal), administración de antibióticos profilácticos durante la cesárea (número de cesáreas en las que se administró antibióticos profilácticos dividido sobre el número de cesáreas) y el manejo activo del alumbramiento para la prevención de la hemorragia postparto (número de mujeres con parto vaginal que recibieron oxitocina para el manejo del alumbramiento dividido sobre el número de mujeres con parto vaginal). Las prácticas correspondientes al cuidado del recién nacido seleccionadas fueron: lactancia materna exclusiva (número de neonatos no hospitalizados con lactancia materna exclusiva dividido sobre el número de neonatos no hospitalizados), restricción rutinaria del contacto madre-hijo (número de recién nacidos vivos sanos en alojamiento conjunto dividido sobre el número de recién nacidos vivos sanos), pinzamiento del cordón umbilical después del primer minuto de vida (número de recién nacidos vivos con pinzamiento del cordón umbilical después del primer minuto de vida dividido sobre el total de recién nacidos vivos), administración de vitamina K para prevenir la enfermedad hemorrágica del recién nacido (número de recién nacidos vivos que recibieron vitamina $\mathrm{K}$ durante la primera hora de vida dividido sobre el total de recién nacidos vivos), esquema completo de vacunación en recién nacidos $>2500$ gramos de peso (número de recién nacidos vivos $>2500$ gramos de peso que recibieron vacunación con $\mathrm{BCG}$, polio oral y hepatitis $\mathrm{B}$ dividido sobre el total de recién nacidos vivos $>$ 2500 gramos de peso) y la posición supina del recién nacido para dormir (número total de recién nacidos vivos que estaban en posición supina para dormir al momento del examen físico de egreso dividido sobre el total de recién nacidos vivos).

El análisis de las variables, frecuencias e indicadores de cada una de las prácticas seleccionadas se llevó a cabo con los programas SIP-CLAP versión 1.34 y SPSS versión 11.5 .

\section{RESULTADOS}

Se incluyeron en total 1775 nacimientos ocurridos entre el 1 de julio de 2004 y el 31 de marzo de 2005. Del total de nacimientos, 1019 (57.4\%) ocurrieron en la Clínica Villapilar, 314 (17.7\%) en la Clínica de La Enea y 442 (24.9\%) en la Clínica de La Asunción. Para el análisis de las prácticas de cuidados generales, tamizaje y diagnóstico durante la gestación, se descartaron 80 madres atendidas en la Clínica Villapilar, 18 en la Clínica La Enea y 24 en la Clínica La Asunción, quienes no asistieron a control prenatal. Para el análisis de las demás prácticas, sí se tuvieron en cuenta. Es de anotar que los controles prenatales fueron realizados usualmente en sitios diferentes a los de la atención del parto. Las pacientes atendidas en las Clínicas de La Enea y La Asunción usualmente son controladas en los diferentes centros de salud de Assbasalud, mientras que las pacientes atendidas en la Clínica Villapilar, usualmente son remitidas de otras instituciones prestadoras de servicios de salud de la ciudad y de otros municipios de Caldas y departamentos vecinos. Los resultados se resumen en las tablas 1 y 2. 


\begin{tabular}{|l|c|c|c|}
\hline \multicolumn{4}{|c|}{ Tabla 1. Características de las gestantes atendidas en tres instituciones de Manizales. } \\
\hline Característica & Villapilar & La Enea & La Asunción \\
\hline Edad materna (años)* & $25.2 \pm 7.3$ & $22.8 \pm 5.9$ & $23 \pm 6.3$ \\
\hline Estudios secundarios** & 56.4 & 62.5 & 61.1 \\
\hline Estudios universitarios** & 9.6 & 3.1 & 2.6 \\
\hline Número de controles prenatales* & $5.1 \pm 2.5$ & $5.4 \pm 2.3$ & $5.4 \pm 2.3$ \\
\hline Régimen vinculado** & 55.5 & 65.4 & 52.6 \\
\hline Régimen subsidiado** & 17.6 & 33.5 & 46.4 \\
\hline Régimen contributivo** & 26.1 & 0 & 0.9 \\
\hline
\end{tabular}

* Promedio \pm desviación estándar

** Porcentaje

\section{DISCUISIÓN}

Algunas prácticas clínicas que se han demostrado benéficas, aún no se han implementado o no se utilizan en forma rutinaria; tal es el caso del uso del carné materno perinatal, el tamizaje para sífilis durante la gestación, el diligenciamiento del partograma, la terminación de la gestación luego de 41 semanas para reducir la mortalidad perinatal, el apoyo continuo a la mujer durante el trabajo de parto y parto, el manejo activo del alumbramiento para prevenir la hemorragia postparto, la lactancia materna exclusiva y el pinzamiento del cordón umbilical después del primer minuto. De igual forma, otras prácticas que se han demostrado inefectivas e incluso perjudiciales, todavía se siguen utilizando, como es el caso del enema evacuante durante el trabajo de parto, la posición en decúbito dorsal (supino) en el periodo expulsivo del parto y la restricción del contacto madre-hijo en los recién nacidos sanos.

A pesar de que se ha demostrado que la suplementación con hierro durante la gestación disminuye la incidencia de niveles bajos de hemoglobina materna al final del embarazo y en el momento del parto, ${ }^{4,5}$ y que la suplementación de ácido fólico mejora los niveles de hemoglobina durante la gestación y en el momento del parto ${ }^{5,6}$ no todas las pacientes reciben esta suplementación.

La ocurrencia continuada de sífilis congénita es indicador de servicios inadecuados de cuidado antenatal y pobre calidad de los programas de control de infecciones de transmisión sexual. ${ }^{7}$ A pesar de existir políticas nacionales para aplicar las pruebas de detección antenatal de dicha infección, no se realiza en forma rutinaria. Por el contrario, es mucho mejor la detección de infección urinaria no sintomática mediante citoquímico de orina durante la primera consulta prenatal, tal como lo recomienda la norma técnica gubernamental, ${ }^{8}$ aunque se ha demostrado que el método ideal es realizar un urocultivo en cada trimestre. ${ }^{9}$

En la actualidad, hay suficiente evidencia para recomendar el uso rutinario de sulfato de magnesio en preeclampsia severa; se ha reportado disminución a la mitad del riesgo de que evolucione a eclampsia y una tendencia a disminuir el riesgo de muerte materna. ${ }^{10,11}$ Además, ha demostrado ser superior a otros anticonvulsivantes para este fin. ${ }^{11-14}$ Sin embargo, no se utiliza aun de manera sistemática en la eclampsia y la preeclampsia severa.

La administración de corticosteroides previos al parto prematuro se emplea en forma casi rutinaria en nuestro medio. Resulta efectiva para prevenir el síndrome de dificultad respiratoria y la mortalidad neonatal. ${ }^{15}$ Otros efectos benéficos reportados son disminución de hemorragia intraventricular y enterocolitis necrotizante. ${ }^{16}$

El uso de enemas durante el trabajo de parto depende de la preferencia del médico que atiende a la paciente y de los recursos disponibles. Sin embargo, causan molestias a la paciente e incrementan los costos. Hasta la fecha, no hay suficiente evidencia para evaluar su uso rutinario durante el trabajo de parto. ${ }^{17}$ 


\begin{tabular}{|c|c|c|c|c|}
\hline Tipo de práctica & Práctica & $\begin{array}{l}\text { Villapilar } \\
\text { (n: 1019) }\end{array}$ & $\begin{array}{l}\text { La Enea } \\
(n: 314)\end{array}$ & $\begin{array}{l}\text { La Asunción } \\
\text { (n: 442) }\end{array}$ \\
\hline \multirow{3}{*}{$\begin{array}{l}\text { Cuidado de } \\
\text { la gestación }\end{array}$} & Portar el carné prenatal a la admisión & $\begin{array}{l}649 / 931 \\
(69.7 \%)\end{array}$ & $\begin{array}{l}212 / 214 \\
(99.1 \%)\end{array}$ & $\begin{array}{l}307 / 312 \\
(98.4 \%)\end{array}$ \\
\hline & Suplementación con hierro a las embarazadas & $\begin{array}{l}864 / 924 \\
(93.5 \%)\end{array}$ & $\begin{array}{l}165 / 181 \\
(91.2 \%)\end{array}$ & $\begin{array}{l}257 / 272 \\
(94.5 \%)\end{array}$ \\
\hline & $\begin{array}{c}\text { Supementación con } \\
\text { ácido fólico a las embarazadas }\end{array}$ & $\begin{array}{l}848 / 924 \\
(91.8 \%)\end{array}$ & $\begin{array}{l}162 / 181 \\
(89.5 \%)\end{array}$ & $\begin{array}{l}252 / 272 \\
(92.6 \%)\end{array}$ \\
\hline \multirow{2}{*}{$\begin{array}{l}\text { Detección } \\
\text { sistemática } \\
\text { y diagnóstico }\end{array}$} & Detección sistemática de sífilis gestacional & $\begin{array}{l}765 / 866 \\
(88.3 \%)\end{array}$ & $\begin{array}{l}239 / 256 \\
(93.4 \%)\end{array}$ & $\begin{array}{l}340 / 364 \\
(93.4 \%)\end{array}$ \\
\hline & $\begin{array}{l}\text { Detección sistemática de bacteriuria } \\
\text { asintomática durante la gestación }\end{array}$ & $\begin{array}{l}868 / 888 \\
(98.1 \%)\end{array}$ & $\begin{array}{l}237 / 241 \\
(98.3 \%)\end{array}$ & $\begin{array}{l}341 / 350 \\
(97.4 \%)\end{array}$ \\
\hline \multirow{4}{*}{$\begin{array}{c}\text { Manejo de } \\
\text { los problemas } \\
\text { del embarazo }\end{array}$} & $\begin{array}{l}\text { Sulfato de magnesio para } \\
\text { el tratamiento de la eclampsia }\end{array}$ & $\begin{array}{c}4 / 5 \\
(80 \%)\end{array}$ & 0 & 0 \\
\hline & $\begin{array}{l}\text { Sulfato de magnesio para el tratamiento } \\
\text { de la pre-eclampsiamoderada }\end{array}$ & $\begin{array}{c}5 / 18 \\
(27.8 \%)\end{array}$ & 0 & 0 \\
\hline & $\begin{array}{l}\text { Sulfato de magnesio para el tratamiento } \\
\text { de la pre-eclampsiasevera }\end{array}$ & $\begin{array}{c}29 / 30 \\
(96.7 \%)\end{array}$ & 0 & 0 \\
\hline & $\begin{array}{l}\text { Corticoides antenatales para promover } \\
\text { la maduración fetal en embarazadas } \\
\text { con alto riesgo de parto pretérmino }\end{array}$ & $\begin{array}{l}143 / 156 \\
(91.7 \%)\end{array}$ & $\begin{array}{c}1 / 2 \\
(50 \%)\end{array}$ & $\begin{array}{c}0 / 1 \\
(0 \%)\end{array}$ \\
\hline \multirow{9}{*}{$\begin{array}{l}\text { Atención } \\
\text { del parto }\end{array}$} & Enema evacuante durante el trabajo de parto & $\begin{array}{l}224 / 643 \\
(34.8 \%)\end{array}$ & - & - \\
\hline & $\begin{array}{l}\text { Rasurado perineal de rutina } \\
\text { para la preparación del parto }\end{array}$ & $\begin{array}{l}14 / 643 \\
(2.2 \%)\end{array}$ & - & - \\
\hline & $\begin{array}{l}\text { Diligenciamiento del partograma } \\
\text { durante el trabajo de parto y parto }\end{array}$ & $\begin{array}{l}3 / 645 \\
(0.5 \%)\end{array}$ & - & - \\
\hline & $\begin{array}{l}\text { Posición decúbito dorsal } \\
\text { (supina) en el período expulsivo del parto }\end{array}$ & $\begin{array}{l}666 / 679 \\
(98.1 \%)\end{array}$ & $\begin{array}{l}286 / 298 \\
(96 \%)\end{array}$ & $\begin{array}{l}405 / 427 \\
(94.8 \%)\end{array}$ \\
\hline & Episiotomía rutinaria en primíparas & $\begin{array}{l}194 / 321 \\
(59 \%)\end{array}$ & $\begin{array}{l}70 / 115 \\
(60.9 \%)\end{array}$ & $\begin{array}{l}76 / 149 \\
(51 \%)\end{array}$ \\
\hline & $\begin{array}{c}\text { Terminación de la gestación luego de } \\
41 \text { semanas para reducir la mortalidad perinatal }\end{array}$ & $\begin{array}{c}5 / 14 \\
(35.7 \%)\end{array}$ & $\begin{array}{c}1 / 2 \\
(50 \%)\end{array}$ & $\begin{array}{c}0 / 1 \\
(0 \%)\end{array}$ \\
\hline & $\begin{array}{l}\text { Apoyo continuo para la mujer } \\
\text { durante el trabajo de parto }\end{array}$ & $\begin{array}{l}326 / 965 \\
(33.8 \%)\end{array}$ & $\begin{array}{l}129 / 265 \\
(48.7 \%)\end{array}$ & $\begin{array}{l}152 / 376 \\
(40.4 \%)\end{array}$ \\
\hline & $\begin{array}{l}\text { Administración de antibióticos } \\
\text { profilácticos en la cesárea }\end{array}$ & $\begin{array}{l}316 / 333 \\
(94.9 \%)\end{array}$ & - & - \\
\hline & $\begin{array}{l}\text { Manejo activo del alumbramiento para } \\
\text { prevención de hemorragia postparto }\end{array}$ & $\begin{array}{l}634 / 674 \\
(94.1 \%)\end{array}$ & $\begin{array}{l}233 / 292 \\
(79.8 \%)\end{array}$ & $\begin{array}{l}307 / 418 \\
(73.4 \%)\end{array}$ \\
\hline \multirow{6}{*}{$\begin{array}{l}\text { Cuidado } \\
\text { del recién } \\
\text { nacido }\end{array}$} & Lactancia materna exclusiva & $\begin{array}{l}693 / 939 \\
(73.8 \%)\end{array}$ & $\begin{array}{l}266 / 267 \\
(99.6 \%)\end{array}$ & $\begin{array}{l}412 / 417 \\
(98.8 \%)\end{array}$ \\
\hline & Restricción rutinaria del contacto madre-hijo & $\begin{array}{l}67 / 895 \\
(7.5 \%)\end{array}$ & $\begin{array}{l}1 / 255 \\
(0.4 \%)\end{array}$ & $\begin{array}{l}4 / 333 \\
(1.2 \%)\end{array}$ \\
\hline & $\begin{array}{l}\text { Pinzamiento del cordón umbilical } \\
\text { después del primer minuto de vida }\end{array}$ & $\begin{array}{l}26 / 973 \\
(2.7 \%)\end{array}$ & $\begin{array}{l}143 / 303 \\
(47.2 \%)\end{array}$ & $\begin{array}{l}193 / 430 \\
(44.9 \%)\end{array}$ \\
\hline & Administración de vitamina $\mathrm{K}$ al recién nacido & $\begin{array}{l}915 / 917 \\
(99.8 \%)\end{array}$ & - & - \\
\hline & $\begin{array}{l}\text { Esquema completo de vacunación } \\
\text { en mayores de } 2500 \mathrm{~g}\end{array}$ & $\begin{array}{l}715 / 721 \\
(99.2 \%)\end{array}$ & - & - \\
\hline & Posición supina del recién nacido para dormir & $\begin{array}{c}849 / 849 \\
(100 \%)\end{array}$ & $\begin{array}{l}97 / 103 \\
(94.2 \%)\end{array}$ & $\begin{array}{c}119 / 124 \\
(96 \%)\end{array}$ \\
\hline
\end{tabular}


El rasurado perineal es un procedimiento realizado antes del nacimiento con el fin de disminuir el riesgo de infección si ocurre un desgarro perineal espontáneo o si se realiza episiotomía. Sin embargo, no se han encontrado diferencias entre hacer o no rasurado en cuanto a enfermedad febril materna se refiere. Incluso, se ha reportado menos colonización por bacterias gram negativas en las pacientes que no fueron rasuradas comparadas con las que sí lo fueron. ${ }^{18}$

Es muy bajo el porcentaje de utilización del partograma a pesar de que en la guía práctica para los cuidados del parto normal de la Organización Mundial de la Salud (OMS) se recomienda la evaluación cuidadosa del progreso del trabajo de parto mediante el partograma. ${ }^{19,20}$ El conjunto de medidas tales como identificación de embarazos de alto riesgo, monitoreo cardíaco fetal y monitoreo de la evolución en el partograma ayudan a identificar al feto en riesgo de asfixia perinatal. ${ }^{21}$

La Iniciativa de Mejores Nacimientos (BBI, Better Births Initiative) es una nueva estrategia desarrollada por profesionales de la salud en Sudáfrica y en el ámbito internacional para brindar a las mujeres una mejor calidad de atención en los nacimientos y mejorar los resultados maternos en países de bajos ingresos. ${ }^{22,23}$ A través del uso de investigaciones basadas en la atención real, las opiniones de las gestantes y la evidencia de los estudios clínicos, la iniciativa ha identificado cuatro pautas fáciles de poner en práctica, mediante las cuales la atención será más compasiva y basada en la evidencia, además de ahorrar tiempo y dinero: Alentar a las mujeres a beber suficiente líquido y a comer si tienen apetito, estimular a la pareja, un amigo, familiar o cuidador no profesional a apoyarlas durante el trabajo de parto, interrumpir los procedimientos rutinarios sin beneficios evidentes, en particular si son vergonzosos o incómodos, y la omisión de intervenciones rutinarias sin beneficios evidentes, tales como la atención del parto en posición supina y la episiotomía. Sin embargo, estas prácticas siguen siendo frecuentemente aplicadas en nuestro medio.
La episiotomía rutinaria es una de las intervenciones sin beneficio evidente que deben ser omitidas. ${ }^{23}$ Al comparar la práctica de episiotomía rutinaria con una política restrictiva para su realización, se encontró que la episiotomía restrictiva tiene beneficios; hay menos trauma perineal posterior, menos necesidad de sutura y menos complicaciones; no hay diferencias con respecto a la mayoría de las medidas de dolor y al trauma perineal o vaginal severo. ${ }^{24}$ Recientemente, Fleming y colaboradores,${ }^{25}$ realizaron un interesante estudio en el que compararon la función muscular perineal mediante electromiografía haciendo mediciones anteparto y postparto en una cohorte de mujeres con diferentes condiciones perineales después de dar a luz (periné intacto, desgarros vaginales de primer grado, desgarros de segundo y tercer grado, y cesárea). El embarazo se asocia con una disminución en la fuerza muscular perineal comparado con el estado postparto. El grado en el cual las mujeres mejoraron o no su función muscular perineal después del parto se relacionó con el trauma perineal durante el parto. Después de controlar la paridad, edad materna, peso al nacer y hábito de fumar, el orden de mejor a peor rendimiento de la función muscular perineal fue: nacimiento por cesárea, periné intacto, desgarro perineal de primer grado, desgarro perineal de segundo o tercer grado, y episiotomía. Aunque las pacientes de los demás grupos mejoraron la función muscular hacia los 6 meses postparto, las mujeres con episiotomía tuvieron una pérdida neta de dicha función después del parto.

El uso de episiotomía está disminuyendo. En Estados Unidos se realizó en el 60.1\% de los partos vaginales espontáneos en 1979, comparado con el $32.8 \%$ ( $\mathrm{p}<0.001)$ en $1997 .{ }^{26}$ En la actualidad se considera que las indicaciones para este procedimiento son estado fetal insatisfactorio detectado durante el expulsivo, distocia de hombros y parto vaginal instrumentado. ${ }^{27}$ En nuestro medio, el uso de episiotomía en primíparas todavía es alto; varía entre el 51 y el $61 \%$.

Aproximadamente el 5\% de los bebés nacen postérmino ( $\geq 42$ semanas), ${ }^{28}$ reportándose mayor 
morbilidad y mortalidad perinatal en estos casos. ${ }^{29-}$ ${ }^{30}$ Comparados con los recién nacidos a término, los postérmino tienen una mortalidad 1.5, 1.8 y 2.9 veces mayor a las 41, 42 y 43 semanas respectivamente. ${ }^{31}$ Se recomienda inducción rutinaria del trabajo de parto luego de la semana 41 de gestación. ${ }^{32}$

El acompañamiento materno por parte de la pareja, un familiar u otra persona que no haga parte del equipo de salud es una práctica benéfica que debería ser rutinaria. ${ }^{23}$ Se ha demostrado que las madres con apoyo continuo durante el trabajo de parto y parto requieren menos medicamentos para el alivio del dolor y sus recién nacidos requieren menos maniobras de reanimación cuando se comparan con mujeres atendidas con el cuidado usual. Además, es menos probable que se les realice episiotomía y perciben la maternidad como una experiencia más positiva. ${ }^{33}$ Las políticas institucionales de los centros de atención de mayor nivel de complejidad no permiten la presencia de familiares en las salas de trabajo de parto.

Cuando se realiza cesárea, se recomienda el uso de antibióticos profilácticos, especialmente ampicilina o cefalosporinas de primera generación. ${ }^{34}$ La hemorragia postparto ocupa el primer o segundo lugar de mortalidad materna en los países en desarrollo. Existe suficiente evidencia para recomendar el manejo activo del alumbramiento. Se asocia con menor pérdida de sangre materna, menos hemorragias postparto mayores de $500 \mathrm{ml}$ y menor necesidad de transfusiones. ${ }^{35}$

La lactancia materna le asegura a los bebés una buena salud y mejores resultados en las áreas de neurodesarrollo y psicosociales. ${ }^{36}$ Una posible explicación acerca de los resultados de la utilización exclusiva de la lactancia materna se basa en que en uno de los centros de atención se realizan cesáreas y los recién nacidos son alimentados inicialmente con fórmula hasta que la madre egrese del servicio de recuperación.

Retardar el pinzamiento del cordón umbilical hasta que deje de pulsar permite el tiempo suficiente para que ocurra transfusión placentaria, aportando al recién cerca de un 30\% adicional de volumen sanguíneo y hasta un $60 \%$ más de eritrocitos, ${ }^{37}$ los cuales al destruirse por hemólisis suplen alrededor de $50 \mathrm{mg}$ de hierro a las reservas del niño. ${ }^{38}$ Los estudios clínicos controlados aleatorizados realizados con recién nacidos a término y prematuros han demostrado que el pinzamiento del cordón luego del primer minuto de vida no incrementa el riesgo de policitemia, taquipnea transitoria ni hiperbilirrubinemia. ${ }^{39}$ Por el contrario, se han reportado beneficios con esta práctica, dentro de los que se destacan niveles de hematocrito y de ferritina sérica más altos a los 2 y 3 meses de vida, ${ }^{40}$ menor necesidad de transfusiones debido a anemia o hipotensión arterial en los recién nacidos prematuros, ${ }^{41,42}$ menos casos de hemorragia intraventricular ${ }^{42}$ y permitir el contacto madre-hijo más temprano. ${ }^{43}$

La deficiencia de vitamina K puede causar hemorragia durante la primera semana de vida en recién nacidos previamente sanos, con una incidencia del 0.25 al 1.7\%, y su uso profiláctico se realiza desde $1961{ }^{44}$ El riesgo de muerte súbita en los lactantes que duermen en posición prona es 3 a 9 veces mayor que el de los bebés que duermen en otra posición (supina o en decúbito lateral). En 1992, la Academia Americana de Pediatría recomendó evitar el uso de la posición prona durante el sueño de los lactantes pequeños, y luego de implementar esta práctica la incidencia de muerte súbita disminuyó en forma importante. ${ }^{45}$

\section{CONCLUSIÓN}

Algunas prácticas clínicas que se han demostrado benéficas, deben implementarse en forma rutinaria o incrementarse su uso, mientras que otras prácticas que se han demostrado inefectivas e incluso perjudiciales, deben abolirse. En el primer grupo se encuentran el uso del carné materno perinatal, la tamización para sífilis durante la gestación, el diligenciamiento del partograma, la terminación de la gestación luego de 41 semanas para reducir la mortalidad perinatal, el apoyo continuo a la mujer durante el trabajo de parto y parto, el manejo activo del alumbramiento para prevenir la hemorragia postparto, la lactancia materna exclusiva y el pinzamiento del cordón 
umbilical después del primer minuto. Al segundo grupo corresponden el enema evacuante durante el trabajo de parto, la posición en decúbito dorsal (supino) en el periodo expulsivo del parto y la restricción del contacto madre-hijo en los recién nacidos sanos. Debe implementarse una política restrictiva de episiotomía, evitando realizarla de manera rutinaria en las primíparas.

Además, se deben hacer todos los esfuerzos posibles para que no se sigan presentando las diferencias institucionales con respecto al uso de prácticas clínicas en el cuidado de la gestación, el trabajo de parto y parto, y el recién nacido. Debe mejorarse la calidad de llenado de la historia clínica materno-perinatal para que los indicadores sean más confiables. Al respecto, recomendamos retroalimentar al personal de salud de los centros de atención con la información obtenida en la presente investigación y continuar realizando talleres de reforzamiento en el diligenciamiento de la historia y el uso del partograma.

\section{REFERENCIAS}

1. Festin MR, Lumbiganon P, Tolosa JE, Finney KA, Ba-Thike $\mathrm{K}$, Chipato $\mathrm{T}$, et al. International survey on variations in practice of the management of the third stage of labour. Bull World Health Organ 2003;81:286-91

2. Enkin M, Keirse M, Nelson J, Crowther C, Duley L, Hodnett H, Hofmeyr J. A Guide to Effective Care in Pregnancy and Childbirth. 3rd ed. Oxford University Press, 2000.

3. World Health Organization. Department of Reproductive Health and Research, UNDP/UNFPA/ WHO/World Bank Special Programme of Research, Development and Research Training in Human Reproduction. The WHO Reproductive Health Library $\mathrm{N}^{\circ}$ 5. Geneva 2002.

4. Mahomed K. Suplementación con hierro y folato en el embarazo (Revisión Cochrane traducida). En: The Cochrane Library, Issue 4, 2003. Chichester, UK: John Wiley \& Sons, Ltd.

5. Mahomed K. Iron supplementation in pregnancy (Cochrane Review). En: The Cochrane Library, Issue 2, 2005. Oxford: Update Software.

6. Mahomed K. Folate supplementation in pregnancy (Cochrane Review). En: The Cochrane Library, Issue 2, 2005. Oxford: Update Software.
7. Saloojee H, Velaphi S, Goga Y, Afadapa N, Steen $\mathrm{R}$, Lincetto $\mathrm{O}$. The prevention and management of congenital syphilis: an overview and recommendations. Bull World Health Organ 2004;82:424-30.

8. Ministerio de Salud. República de Colombia. Norma técnica para la detección temprana de las alteraciones del embarazo. Resolución 00412 de 2000.

9. McIsaac W, Carroll JC, Biringer A, Bernstein P, Lyons E, Low DE, et al. Screening for asymptomatic bacteriuria in pregnancy. J Obstet Gynaecol Can 2005;27:20-4.

10. Sibai BM. Magnesium sulfate prophylaxis in preeclampsia: evidence from randomized trials. Clin Obstet Gynecol 2005;48:478-88.

11. Duley L, Gülmezoglu AM, Henderson-Smart DJ. Magnesium sulphate and other anticonvulsants for women with pre-eclampsia (Cochrane Review). En: The Cochrane Library, Issue 2, 2005. Oxford: Update Software.

12. Duley L, Henderson-Smart D. Sulfato de magnesio versus diazepam para la eclampsia (Revisión Cochrane traducida). En: The Cochrane Library, Issue 4, 2003. Chichester, UK: John Wiley \& Sons, Ltd.

13. Duley L, Henderson-Smart D. Magnesium sulphate versus phenytoin for eclampsia (Cochrane Review). En: The Cochrane Library, Issue 2, 2005. Oxford: Update Software.

14. Duley L, Gulmezoglu AM. Sulfato de magnesio versus cóctel lítico para la eclampsia (Revisión Cochrane traducida). En: The Cochrane Library, Issue 4, 2003. Chichester, UK: John Wiley \& Sons, Ltd.

15. Crowley P. Prophylactic corticosteroids for preterm birth (Cochrane Review). En: The Cochrane Library, Issue 2, 2005. Oxford: Update Software.

16. Dudley DJ, Waters TP, Nathanielsz PW. Current status of single-course antenatal steroid therapy. Clin Obstet Gynecol 2003;46:132-49.

17. Cuervo LG, Rodríguez MN, Delgado MB. Enemas during labor. En: The Cochrane Library, Issue 2, 2005. Chichester, UK: John Wiley \& Sons, Ltd.

18. Basevi V, Lavender T. Routine perineal shaving on admission in labour (Cochrane Review). En: The Cochrane Library, Issue 2, 2005. Oxford: Update Software.

19. Maternal Health and Safe Motherhood Programme. Division of Family Health. World Health Organization. Preventing prolonged labour: a practical guide. The partograph. Parts 1 to 4 . WHO/FHE/MSM/93.8. 
20. Grupo de Trabajo Ténico. Organización Mundial de la Salud. Cuidados en el parto normal: una guía práctica. Rev Hosp Mat Inf Ramón Sardá 1999;18:78-80.

21. Saloojee H. Prevención de la morbimortalidad en recién nacidos a término con asfixia perinatal: Aspectos prácticos de la BSR (última revisión: 1 de diciembre de 2001). La Biblioteca de Salud Reproductiva de la OMS, $\mathrm{N}^{0}$ 7. Oxford: Update Software Ltd; 2004.

22. Smith H, Brown H, Hofmeyr GJ, Garner P. Evidence based obstetric care in South Africa --influencing practice through the "Better Births Initiative". S Afr Med J 2004;94:117-20.

23. Una introducción a la Implementation aids: "Iniciativa Mejores Nacimientos". La Biblioteca de Salud Reproductiva de la OMS, $N^{\circ} 7$, Ginebra, Update Software Ltd, Oxford, 2004.

24. Carroli G, Belizan J. Episiotomy for vaginal birth (Cochrane Review). En: The Cochrane Library, Issue 2, 2005. Oxford: Update Software.

25. Fleming N, Newton ER, Roberts J. Changes in postpartum perineal muscle function in women with and without episiotomies. J Midwifery Womens Health 2003;48:53-9.

26. Weber AM, Meyn L. Episiotomy use in the United States, 1979-1997. Obstet Gynecol 2002;100:1177-82.

27. Cleary-Goldman J, Robinson JN. The role of episiotomy in current obstetric practice. Semin Perinatol 2003;27:3-12.

28. Shea KM, Wilcox AJ, Little RE. Postterm delivery: a challenge for epidemiologic research. Epidemiology 1998;9:199-204.

29. Divon MY, Haglund B, Nisell H, Otterblad PO, Westgren M. Fetal and neonatal mortality in the postterm pregnancy: the impact of gestational age and fetal growth restriction. Am J Obstet Gynecol 1998;178:726-31.

30. Hollis B. Prolonged pregnancy. Curr Opin Obstet Gynecol 2002;14:203-7.

31. Olesen AW, Westergaard JG, Olsen J. Perinatal and maternal complications related to postterm delivery: a national register-based study, 1978-1993. Am J Obstet Gynecol 2003;189:222-7.

32. Crowley P. Interventions for preventing or improving the outcome of delivery at or beyond term (Cochrane Review). En: The Cochrane Library, Issue 2, 2005. Oxford: Update Software.
33. Hodnett ED, Gates S, Hofmeyr G J, Sakala C. Continuous support for women during childbirth (Cochrane Review). En: The Cochrane Library, Issue 2, 2005. Oxford: Update Software.

34. Smaill F, Hofmeyr GJ. Antibiotic prophylaxis for cesarean section (Cochrane Review). En: The Cochrane Library, Issue 2, 2005. Oxford: Update Software.

35. Prendiville WJ, Elbourne D, McDonald S. Active versus expectant management in the third stage of labour (Cochrane Review). En: The Cochrane Library, Issue 2, 2005. Oxford: Update Software.

36. Gartner LM, Morton J, Lawrence RA, Naylor AJ, O'Hare D, Schanler RJ, et al. American Academy of Pediatrics. Section on Breastfeeding. Breastfeeding and the use of human milk. Pediatrics 2005;115:496-506.

37. Yao AC, Moinian M, Lind J. Distribution of blood between infant and placenta after birth. Lancet 1969;2:871-3.

38. World Health Organization. Care in normal birth: report of the technical working group meeting in normal birth. March 25-29, 1996. Geneve: World Health Organization, Maternal Health and Safe Motherhood Program; 1996.

39. Arango F, Mejía JC. ¿Cuándo pinzar el cordón umbilical? Rev Colomb Obstet Ginecol 2004;55:136-45.

40. Geethanath RM, Ramji S, Thirupuram S, Rao YN. Effect of timing of cord clamping on the iron status of infants at 3 months. Ind Pediatr 1997;34:103-6.

41. Ibrahim HM, Krouskop RW, Lewis DF, Dhanireddy R. Placental transfusion: umbilical cord clamping and preterm infants. J Perinatol 2000;20:351-4.

42. Rabe H, Reynolds G, Diaz-Rossello J. Early versus delayed umbilical cord clamping in preterm infants (Cochrane Review). En: The Cochrane Library, Issue 2, 2005. Oxford: Update Software.

43. Oxford Midwives Research Group. A study of the relationship between the delivery to cord clamping interval and the time of cord separation. Midwifery 1991;7:167-76.

44. American Academy of Pediatrics. Committee on Fetus and Newborn. Controversies concerning vitamin K and the newborn. Pediatrics 2003;112:191-2.

45. Paris CA, Remler R, Daling JR. Risk factors for sudden infant death syndrome: changes associated with sleep position recommendations. J Pediatr 2001;139:771-7. 